\title{
THE VOYAGER MISSIONS TO THE OUTER SYSTEM
}

\author{
E. C. STONE, Voyager Project Scientist \\ California Institute of Technology, Pasadena, Calif. 91125, U.S.A.
}

(Received 24 May, 1977)

The twelve papers comprising this issue provide a review of the breadth of the scientific investigations which will be undertaken by the NASA Voyager Project. The objectives of the Voyager Missions are to conduct exploratory investigations of the Jupiter and Saturn planetary systems and of the interplanetary medium from Earth to Saturn. If all goes well, an extended mission will provide the first flyby investigation of Uranus and its newly discovered rings and interplanetary studies beyond $20 \mathrm{AU}$. Emphasis will be placed on comparative studies of the planets and satellites, on studies of the rings of Saturn and Uranus, and on interplanetary and interstellar studies at increasing distances from the Sun.

The diversity of the scientific instrumentation described in the following papers is just one indication of the breadth of the planned investigations. Another indication is the involvement of 100 scientists from 38 institutions as co-investigators or coauthors on the investigation papers.

Equally important indications are the advanced capability of the spacecraft and the variety of unique observational opportunities afforded by the mission design, as described in the first paper. These opportunities include close flybys of the four Galilean satellites and of six Saturnian satellites, passage through the magnetic flux tube connecting Io to Jupiter, a very close encounter with Titan, Sun and Earth occultations by Jupiter, Saturn, and Titan, an Earth occultation by Saturn's Rings, and a high latitude passage at Saturn with the option of continuing to Uranus and possibly Neptune.

One of the major purposes of the following detailed descriptions of the Voyager objectives and investigations is to provide impetus and focus for theoretical and observational efforts of those individuals in the scientific community with related interests. Over the next ten years the Voyager Mission should provide many clues to understanding the Solar System, just as Galileo did 367 years ago when he first turned his telescope toward the celestial sphere. 